\title{
ArtigoOrigne
}

\section{RESPOSTAS METABÓLICAS E CARDIORRESPIRATÓRIAS AO EXERCíCIO MÁXIMO E SUBMÁXIMO EM MENINAS EUTRÓFICAS E COM DESNUTRIÇÄO PREGRESSA}

*A.C. Franandez;I.P. Gntra;A.L Samaya;M. Fisgerg;A.C. da Silva

Departamento dePediatria eN eurofisiologia eFisiologia Endócrina -UniversidadeFederaldeSão Paulo; Departamento deN utrição-UniversidadeFederaldeO uro Preto-M G

RESUMO - Objetivo: verificar se a desnutrição pregressa, além de causar déficit na estatura de meninas também poderia estar causando uma limitação funcional à capacidade de realização de exercício máximo e submáximo em bicicleta ergométrica.

Casuistica. 24 meninas, 12 eutróficas de idade (média \pm DP) $9,13 \pm 0,79$ anos; peso $27,64 \pm 3,64 \mathrm{~kg}$; ealtura $131,31 \pm 6,04 \mathrm{~cm}$; e 12 com desnutrição pregressa; idade 9,75 $\pm 1,10$ anos; peso $25,16 \pm 2,33 \mathrm{~kg}$; e altura $125,06 \pm 3,90 \mathrm{~cm}$.

Metodo LOGIA. Avaliação clínica e teste ergoespirométrico em bicicleta ergométrica, a partir do qual foram obtidas as variáveis metabólicas ecardiorrespiratórias analisadas. Protocolo de teste: 2 minutos iniciais a 25 watts, seguidos de incrementos de 15 watts a cada 2 minutos, até a exaustão.

RESULTADOS. Testes de esforço máximo: não foram observadas diferenças entre o grupo de meninas eutróficas (E) e 0 grupo de meninas com desnutrição pregressa (D) para nenhuma das variáveis analisadas: $\mathrm{VO}_{2 \text { max }} / \mathrm{min}_{1}, \mathrm{VO}_{2_{\text {max }}} \mathrm{ml} / \mathrm{min} / \mathrm{kg}_{1}, \mathrm{VE}_{\text {max }}, \mathrm{R}_{1} \mathrm{FC}_{\text {max }}$ $\% \mathrm{FC}_{\text {max }}$ prev e Watts max. Teste de esforço submáximo: foram observadas diferençasentre os grupos para as seguintes variáveis: $\mathrm{VO}_{2} / /$ min LA, \% VO 2max $_{\text {max }}, F C L A, \% \mathrm{FC}_{\text {max }}$ LA, VE LA, \% VE $\mathrm{Eax}_{\text {max }} \mathrm{LA}$ $\mathrm{Não}$ foram encontradas diferenças entre os grupos para: $\mathrm{VO}_{2} \mathrm{ml} /$ $\mathrm{min} / \mathrm{kg}$ LA e Watts LA.

CONCLUSÄ0 0 restabelecimento da relação peso para estatura em meninas com desnutrição pregressa, considerado como critério de eutrofia, é válido também para indicar ausência de limitação funcional da capacidade de realização de exercício.

Unitermos: Exercício. Consumo de oxigênio. Meninas. Desnutrição pregressa. Eutrofia.

\section{INTRODUÇão}

Um dosproblemasmaiscaracterísticos relacionados à nutrição é a desnutrição, pois a privação dos nutrientes essenciais nos primeiros anos de vida pode levar à diminuição davelocidade do crescimento e do desenvolvimento, causando um tipo de desnutrição conhecida por proteico-calórica. Este tipo é a mais comum e está associadaaelevadas taxas de morbidadee mortalidade (Chaves, 1985).

0 Brasil apresenta um grande contingente de desnutridos, emboratenhaevoluído favoravelmentedadécadade 70 paraos

*Correspondência:

R. Pedro de Toledo, 980 -conj. 51 -Vila Clementino-Cep:04039-002 São Paulo/ SP-Brasil-Tel.: (11)5575-3875 E-mail:anacf@osite.com.br dias atuais. O sdados da pesquisa nacional desenvolvida pelo IN AN e pelo IBGE, em Março de 1990, indicam que $31 \%$ das crianças brasileiras menores de cinco anos apresentam algum tipo de desnutrição, sendo que $5 \%$ sofrem de desnutrição moderada ou grave. 0 Sudeste, emboranão apresente o maior índice de prevalência de desnutrição, concentra o segundo contingentededesnutridos.

N os países em desenvolvimento o retardo no crescimento, provocado peladesnutrição, pode afetar até $50 \%$ das crianças menores de cinco anos (Avelar et al., 1991). N o Brasil, segundo pesquisarealizada em 1989 (PN SN , 1989), a prevalência de déficitde estatura/idadeem crianças préescolaresfoide 15,4\% . Durante ainfância adesnutrição crônica, ealgumas doenças, atrasam o crescimento reduzindo o desenvolvimento ósseo e retardando a puberdade (Meredith \& D wyer, 1991).

A adaptação àbaixaingestão de nutrientes, condições sanitárias deficientes, e/ou doenças da infância, são freqüentemente caracterizadas por um menor potencial de crescimento, tornando os indivíduos menores do que a suadeterminação genética (Greulich, 1958; Satynarayanaet al., 1986).

U m estudo realizado em favelas de São Paulo mostrou elevada correlação entrea obesidadenosadolescenteseadesnutrição pregressa (Saw ayaet al., 1995). A obesidade e o sobrepeso associados com adesnutrição pregressa, foram encontrados em $8,7 \%$ dos meninos e $7,5 \%$ das meninas, enquanto que em crianças com estatura normal para idade 3,7\% daquelese $4,7 \%$ 
daquelas apresentaram obesidade. N os adolescentes a obesidade foi identificada em 35\% das meninas com baixa estatura paraaidade, enquanto quenas com estatura normal para idade (segundo N CHS ) a prevalênciade obesidade só foi identificada em $13 \%$. De acordo com os dados apresentados, parece que as meninas são mais afetadas pela obesidade quando comparadasaosmeninos.

De acordo com Sawaya; Amigo \& Sigulem (1989) as crianças com desnutrição pregressa, sem déficit de peso paraaidade, são consideradas eutróficas. Em estudo maisrecente com crianças com desnutrição pregressa, observou-se que estas apresentavam valores mais baixos para a taxa de metabolismo de repouso, expressos por calorias por dia, evalores maisaltosquando expressos por quilograma de peso corpóreo, e massa magraquando comparadas acriançaseutróficas, alteração tipicamente descritaparaas desnutridas (Grilo, 1996).

A desnutrição causadiminuição damassa muscular epodeinfluenciar o VO ${ }_{2 \text { máx }}$ (Spurr \& Reina, 1990; Armstrong\& W esman, 1994). $Q$ uando o consumo de oxigênio éexpresso em termosdemassacorporal magra, a diferençaentre desnutridoseeutróficosédrasticamentereduzidae, em algunscasos, ocorre umainversão. Indivíduosdesnutridospodem apresentar valores significativamente mais elevadosdo que oseutróficos, provavelmenteassociadosaumamenor contribuição de gorduraparao peso do músculo do desnutrido (Spurr \& Reina, 1990; Armstrong \& Wesman, 1994).

Segundo estudos realizado sem cortadores de cana, em 1983, existe uma relação entre estado nutricional e produtividade, levando à especulação sobre os efeitosque adesnutrição crônicateria, duranteainfância, no desenvolvimento do consumo máximo de oxigênio $\left(\mathrm{VO}_{2 \text { máx }}\right)$, o principalíndice de aptidão cardiorrespiratória, bem como no possível prognóstico dascapacidades do adulto serem influenciadaspelacriançaafetada. Foi observado que adultos com diferentes níveis de nutrição mostraram uma redução progressivano consumo máximo de $\mathrm{O}_{2}$, de acordo com a severidade da desnutrição, relacionadacom diferençasna massamagra (Spurr et al., 1983).

Em um estudo realizado com crianças declasse socioeconômicabaixa, no interior de São Paulo, com deficiências denutrição, observou-se que estas crianças apresentavam atrasos no desenvolvimento e pior performance física do que crianças eutróficas damesmaregião, provavelmente devido a um quadro de desnutrição vigente (Desai et al., 1981).

$\mathrm{N}$ ão foi possível encontrar na literatura dadosa respeito de exercício em crianças com desnutrição pregressa. U mavez que estácomprovado queestetipo de desnutrição causa déficit em relação à estatura, torna-se importante verificar se houvetambém prejuízo no que diz respeito àcapacidade normal paraarealização de exercícios estimada pelo VO ${ }_{2 \max }$ e limiar anaeróbio, e se, ao alcançar uma relação peso/estatura esperada, a criança passaa ap resentar também respostasfisiológicas ao exercício semelhantesàscrianças eutróficas.

0 objetivo desteestudo foi comparar as respostasmetabólicas e cardiorrespiratórias ao exercício máximo e submáximo de meninas eutróficas e com desnutrição pregressa e verificar se o déficit de estatura, resultado dadesnutrição pregressa, causa algum tipo de influência ou de prejuízo na capacidade funcional da realização do teste de esforço máximo ou submáximo.

\section{Metodologias}

Casuística:

A amostrafoi constituídade 24 crianças do sexo feminino pré-púberes, poismeninas apresentam uma maior prevalência para a obesidade, segundo estudo realizado em
São Paul07 , nafaixaetáriados9 aos 11 anos, residentesem favelas dacidadede São Paulo. Dozemeninas com peso eestaturanormais - valores na faixa de 90 a $110 \%$ dos valores referênciaespecífico paraaidade-eestatural idade com 95\% de adequação (NCHS, 1973), outras 12 com peso para estatura normal (90-110\% dosvalores dereferência - NCHS), mas com os critérios para serem classificadasparadesnutrição pregressa, definidacomo abaixo de $95 \%$ deestaturaesperada para aidade, de acordo com o critério de Watelow (Waterlow, 1976, 1977).

Todas as crianças foram submetidas à uma avaliação clínica, esomente foram incluídas aquelas que não apresentaram algumapatologia préviaenem contraindicação para atividade física intensa. 0 consentimento para a participação no estudo foi obtido dos pais ou responsáveis pelas crianças, que foram informados de todos os procedimentos, tendo liberdade parainterromper a participação em qualquer momento da pesquisa.

Esteestudo foi aprovado pelaC omissão deÉticaMédicadaU niversidadeFederal de São Paulo - Escola Paulista de Medicina (UN IFESP - EPM).

Avaliação antropométrica do crescimento:

Peso: 0 peso corporal foi obtido através de balança eletrônica, marca Kratos, com capacidade máxima para 150 kg e subdividida em $50 \mathrm{~g}$ (Grilo, 1996).

Estatura: A estaturafoi verificada com fita métrica (Microtoise - Stanley - Mabo $L t d)$, um instrumento simples, com extensão de 2,00 m, dividida em centímetrose subdividida em milímetros, com visor de plástico e esquadro acoplado a uma das extremidades (Grilo, 1996).

\section{TESTE ERGOESPIROMÉTRICO}

0 teste incremental deesforço máximo foi realizado em cicloergômetro compatível comaalturadascrianças. 0 início daativida- 
Fernandez Ac et al.

de foi com 25 watts, com o primeiro aumento de carga de 10 watts, seguidos de aumentos de 15 wattsacada 2 minutosaté à exaustão, ou 0 alcance de um ou mais critérios de obtenção do V0 ${ }_{2 \text { máx }}$ (ACSM, 1994).

Durante o teste foram obtidas variáveis metabólicase cardiorrespiratórias através de um sistema metabólico computadorizado.

Asvariáveis respiratórias e metabólicas foram obtidaspelo método de calorimetria indiretarespiratória, por meio de um sistemametabólico (VistaX T metabolic sistem, EUA) computadorizado (Intel 486, DX2, 66mhz).

A monitorização, aquisição eregistro da freqüência cardíacafo i feita através de um freqüencímetro polarVANTAGEXL, acada 5 segundos, durante todo o teste. Para definição dosvalores dafreqüênciacardíaca do limiar anaeróbio foi utilizadaa médiados quatro valores dafreqüência cardíaca correspondente ao tempo do limiar anaeróbio. Paraa definição dafreqüência cardíacamáximafoi utilizado o valor máximo registrado pelo aparelho.

\section{Limiar Anaeróbio}

Foi determinado pelo aumento no $\mathrm{VO}_{2}$ eVE $/ \mathrm{VO}_{2}$, sem, no entanto, haver aumento na relação $\mathrm{VE} / \mathrm{VCO}_{2}$, e as trocas gasosas respiratórias apresentarem um valor de 1 ou maior que este (Zeballos \& W eisman, 1994).

\section{Métodos estatísticos:}

Paraaanálise dos resultadosforam aplicados os seguintes testes:

1. Teste t de student para a análise de variáveisindependentes(Sokal, 1969), para comparar o peso e a estatura entre as crianças eutróficas (G rupo I) e as crianças com desnutrição pregressa(Grupoll).

2. Teste de Mann-W hitney (Siegel, 1975)
Tabela 1- Número $(\mathrm{N})$, idade (anos), peso $(\mathrm{kg})$ e altura $(\mathrm{cm})$ de meninas eutróficas (Grupo I) e com desnutrição pregressa (Grupo II).

\begin{tabular}{ccccc}
\hline & $\mathbf{N}$ & Idade & Peso & Altura \\
$\mid$ & 12 & $9,13 \pm 0,79$ & $27,64 \pm 3,64$ & $131,31 \pm 6,04$ \\
$\|$ & 12 & $9,75 \pm 1,10$ & $25,16 \pm 2,33$ & $125,06 \pm 3,90$ \\
& & $z=-1,674$ & $t=1,966$ & $t=2,972 *$ \\
\hline $\mathbf{p}$ & & ns & ns & $\mathbf{0 , 0 5}$ \\
\hline
\end{tabular}

Tabela 2 - Peso esperado para a idade (P E) e a relação peso para a estatura (P/E) de meninas eutróficas (Grupo I) e com desnutrição pregressa (Grupo II).

\section{P E}

$27,98 \pm 3,90$

$24,22 \pm 2,08$
P/E

$101,09 \pm 9,49$

$105,37 \pm 8,59$
Tabela 3 - Estatura esperada para a idade (E/E) e a relação estatura para a idade (E/I) de meninas eutróficas (Grupo I) e com desnutrição pregressa (Grupo II).

\begin{tabular}{|c|c|c|}
\hline & $E / E$ & $E / I$ \\
\hline । & $134,98 \pm 5,03$ & $97,36 \pm 5,06$ \\
\hline$\|$ & $140,63 \pm 6,80$ & $89,08 \pm 3,95$ \\
\hline \multicolumn{3}{|c|}{$\begin{array}{l}\text { Tabela } 4 \text { - Consumo máximo de oxigênio dividido por quilogramas de peso corporal } \\
\text { (V02maxmL/min/kg) medido em litrospor minuto (V02maxL/min) e para meninas } \\
\text { eutróficas(Grupo l) e com desnutrição pregressa (Grupo II). }\end{array}$} \\
\hline \multirow{4}{*}{$\begin{array}{l}\text { I } \\
\text { II }\end{array}$} & $\mathrm{V} 02 \mathrm{maxL} / \mathrm{min}$ & V02maxmL/min/kg \\
\hline & $1,11 \pm 0,25$ & $40,23 \pm 6,98$ \\
\hline & $1,02 \pm 0,19$ & $40,53 \pm 6,32$ \\
\hline & $z=-0,69$ & $z=0,00$ \\
\hline$p$ & ns & ns \\
\hline
\end{tabular}

Tabela 5 - Ventilação máxima (VEmax) medida em litros por minuto e Q uociente de trocasventilatórias ( $R$ ) durante teste de esforço máximo de meninas eutróficas (Grupol) e com desnutrição pregressa (Grupo II).

\begin{tabular}{ccc}
\hline & VEmax & $\mathbf{R}$ \\
$\mid$ & $40,61 \pm 8,79$ & $1,13 \pm 0,07$ \\
$\|$ & $38,25 \pm 8,95$ & $1,13 \pm 0,05$ \\
& $z=-0,577$ & $z=-0,115$ \\
\hline $\mathbf{p}$ & ns & ns \\
\hline
\end{tabular}


paracomparar as crianças dosgrupos lell paraas demaisvariáveis estudadas.

Foifixado o $\alpha$ em 0,05 ou $5 \%(\alpha \leq 0,05) 0$ nível de rejeição da hipótese de nulidade, assinalando-se com um asterisco osvalores significativos.

\section{Resultados}

Antropometriadasmeninaseutróficase com denutrição pregressa.

0 sresultados deidade, peso e estatura das meninas eutróficas e com desnutrição pregressa estão apresentados na tabela 1 como média \pm desvio padrão:

$N$ ão houve diferençaestatisticamente significativa paraidadee peso entre os dois grupos, porém para o quesito alturaaconteceu o oposto, umavez que os gruposforam selecionados com nível de cortealtura/idade de $95 \%$, o quecaracterizaa desnutrição pregressa(Waterlow,1977).

O sresultados de peso esperados paraa estatura e relação peso paraa estatura das meninas eutróficas e com desnutrição pregressaestão apresentados natabela 2 como média desvio padrão. 0 s resultados de estaturaesperados paraaidade e arelação estatura paraaidadeestão apresentadosna tabela 3 como média desvio padrão:

0 s valores de peso para a estatura e desta paraaidade estão de acordo com os níveis de corte descritosnametodologia.

\section{Resultados do teste de esforço máximo}

Os valores de consumo máximo de oxigênio no teste de esforço máximo para meninas eutróficas e com desnutrição pregressaestão apresentados natabela 4 como média desvio padrão:

$N$ ão houve diferençasignificativa entre osgrupos paraosvalores de consumo máximo de oxigênio, tanto em litros por minuto quanto dividido por quilogramade peso corporal.

O svalores máximos de ventilação e do quociente dastrocas respiratórias durante 0
Tabela 6 - Freqüência cardíaca máxima (FCmax) e porcentagem da freqüência cardíaca máxima prevista (\% FCmax prev) medidasem batimentos por minuto de meninas eutróficas (Grupo I) e com desnutrição pregressa (Grupo II).

\begin{tabular}{ccc}
\hline & FCmax & \% FCmax prev \\
I & $195,33 \pm 9,13$ & $92,87 \pm 4,33$ \\
1 & $193,85 \pm 11,18$ & $92,80 \pm 4,87$ \\
& $\mathbf{z}=-0,202$ & $\mathrm{z}=-0,548$ \\
\hline $\mathbf{p}$ & ns & ns \\
\hline
\end{tabular}

Tabela 7 - Carga máxima de trabalho (Watts max) expressa em watts de meninas eutróficas (Grupol) e com desnutrição pregressa (Grupo II).

\begin{tabular}{cc}
\hline & Watts max \\
$\mid$ & $90,00 \pm 20,56$ \\
$\|$ & $91,25 \pm 11,33$ \\
& $z=-0,663$ \\
\hline $\mathbf{p}$ & ns \\
\hline
\end{tabular}

Tabela 8 - Consumo de oxigênio, no limiar anaeróbio ventilatório (LAV), dividido por quilogramas de peso corporal (V02mL/min/kg LAV), medido em litros por minuto (V02L/min LAV) e porcentagem do consumo máximo de oxigênio (\%V02max) para meninas eutróficas (Grupo I) e com desnutrição pregressa (Grupo II).

\begin{tabular}{cccc}
\hline & VO2mL/min/kg LAV & V02L/min LAV & $\%$ V02max \\
I & $28,06 \pm 4,87$ & $0,77 \pm 0,14$ & $70,07 \pm 7,52$ \\
$\|$ & $24,81 \pm 5,31$ & $0,62 \pm 0,13$ & $61,21 \pm 9,83$ \\
& $z=-0,443$ & $z=-2,424 *$ & $z=-2,309 *$ \\
\hline $\mathbf{p}$ & ns & $\mathbf{0 , 0 5}$ & $\mathbf{0 , 0 5}$ \\
\hline
\end{tabular}

Tabela 9 - Freqüência cardíaca no limiar anaeróbio ventilatório (FC LAV) e porcentagem da freqüência cardíaca máxima no limiar anaeróbio ventilatório (\% FCmax LAV) medidas em batimentos por minuto de meninas eutróficas (Grupo I) e com desnutrição pregressa (Grupo II).

\begin{tabular}{cccc}
\hline & FC LAV & \%FCmax LAV \\
& \multicolumn{1}{c}{$166,58 \pm 14,25$} & $85,31 \pm 6,41$ \\
& 1 & $146,33 \pm 17,40$ & $75,50 \pm 7,73$ \\
& $z=-2,915^{*}$ & $\mathrm{z}=-2,771^{*}$ \\
\hline $\mathbf{p}$ & $\mathbf{0 , 0 1}$ & $\mathbf{0 , 0 1}$ \\
\hline
\end{tabular}


teste de esforço máximo estão apresentados na tabela 5 com valores expressos como média desvio padrão.

$N$ ão houve diferença estatisticamente significativaentre osgrupos para os valores deventilação máximae quociente respiratório.

A tabela 6 apresenta os valores de freqüência cardíacamáxima obtida durante 0 teste de esforço e asua porcentagem prevista em valores expressos como média \pm desvio padrão:

O s grupos I e ll não apresentaram diferenças significativas para os valores de freqüência cardíaca máxima e para a sua porcentagem esperadadurante o teste deesforço.

A tabela 7 apresentaosvalores de carga máxima de trabalho alcançada durante 0 teste de esforço de meninas eutróficas e com desnutrição pregressa expressa em média \pm desvio padrão.

$\mathrm{N}$ ão houve diferenças entre os dois grupos para os valores de carga máxima atingidadurante 0 teste deesforço.

Resultados submáximos do teste de esforço

O svalores de consumo de oxigênio no limiar anaeróbio e daporcentagem do consumo máximo de oxigênio de meninas eutróficase com desnutrição pregressaestão apresentados na tabela 8 e os valores estão expressos como média desvio padrão.

N ão houve diferençaestatísticasignificativaentre osdoisgrupos para os valores de consumo de oxigênio dividido por quilogramade peso corporal no limiar anaeróbio ventilatório. Asmeninaseutróficasapresentaram maiores valores parao consumo de oxigênio em litrosno limiar anaeróbio ventilatório e para a porcentagem do $\mathrm{VO}_{2 \max }$ utilizadano limiar anaeróbio ventilatório.

A tabela 9 apresenta os valores de freqüênciacardíacaobtidano limiar anaeróbio ventilatório durante o teste de esforço ea

Tabela 10 - Ventilação no limiar anaeróbio ventilatório (VE LAV) medida em litros por minuto e porcentagem da ventilação máxima no limiar anaeróbio ventilatório medida em litros por minuto (\%VEmax LAV) de meninas eutróficas (Grupo I) e com desnutrição pregressa (Grupo II).

\begin{tabular}{ccc}
\hline & VE LAV & \% VEmax LAV \\
$\mid$ & $24,18 \pm 5,81$ & $59,64 \pm 8,45$ \\
$\|$ & $17,69 \pm 4,30$ & $46,95 \pm 10,59$ \\
& $z=-2,713^{*}$ & $z=-2,655^{*}$ \\
\hline $\mathbf{p}$ & $\mathbf{0 , 0 1}$ & $\mathbf{0 , 0 1}$ \\
\hline
\end{tabular}

Tabela 11 - Carga de trabalho no limiar anaeróbio ventilatório (Watts LAV) expressa em watts de meninas eutróficas (Grupo I) e com desnutrição pregressa (Grupo II).

\begin{tabular}{cc}
\hline Watts & LAV \\
$\mid$ & $51,25 \pm 17,47$ \\
$\|$ & $45,00 \pm 9,77$ \\
& $z=-0,663$ \\
\hline $\mathbf{p}$ & ns \\
\hline
\end{tabular}

sua porcentagem no limiar anaeróbio ventilatório com os valores expressos como média \pm desvio padrão.

Asmeninas eutróficasapresentarammaiores valores para a freqüência cardíaca no limiar anaeróbio ventilatório e paraaporcentagem dafreqüênciacardíacamáximautilizadano limiar anaeróbio ventilatório.

Osvaloresdaventilaçãonolimiaranaeróbioventilatórioedaporcentagemdaventila çãomáximanolimiaranaeróbioventilatório duranteotestedeesforçomáximoestãoapresentadosnatabela10comvaloresexpressos comomédia desviopadrão.

As meninas eutróficas apresentaram maiores valores para a ventilação no limiar anaeróbio ventilatório e para a porcentagem daventilação máximautilizadano limiar anaeróbio ventilatório.

A tabela 11 apresenta os valores de cargade trabalho no limiar anaeróbio ventilatório de meninas eutróficas e com desnutrição pregressaexpressaem médiades- vio \pm padrão.

0 grupo l, formado por meninas eutróficas, não apresentou valores de carga de trabalho diferentes do grupo II, meninas com desnutrição pregressa.

\section{Discussão}

Segundo os dados das tabelas 1, 2 e 3 , as crianças do presente estudo estão dentro dospadrões paraserem classificasnos dois gruposdescritosnametodologia, um denominado de eutróficas e o outro, de grupo com desnutrição pregressa.

$Q$ uando o grupo das eutróficasé comparado aos dadosnacionais apresentados por França, Matsudo, França(1993) percebe-se que os dados so bre estatura e peso são coincidentes, entretanto o grupo das desnutridas pregressas apresenta menor valor para a estatura, enquanto os valores depeso são coerentes com os descritosno citado estudo. Estadiferençana estaturaéa 
principal característica da desnutrição pregressa.

O sestudos existentesutilizando amesma metodologia ou semelhante a deste estudo, medidasindiretas atravésda análise de gases expirados, são, na sua maioria, com criançasatletas, crianças que apresentam algum tipo de patologia, ou devido a circunstâncias pós-operatórias. Poucossão osestudos com as crianças não-atletas e os estudos realizados com as desnutridas são aindamais escassos.

Cooper; Weiler-Ravell; Whipp \& Wasserman (1984) reportaram valores de $\mathrm{VO}_{2_{\text {max }}}(\mathrm{mL} / \mathrm{min} / \mathrm{kg})$ com média de $38 \pm 7$ parameninas não-atletas com idade média de nove anos, enquanto que Armon; Cooper; Flores; Zanconato \& Barstow, (1991) relataram médiaigual a $43 \pm 6(\mathrm{~mL} /$ $\mathrm{min} / \mathrm{kg}$ ), paracrianças (meninosemeninas) não-atletas com idades entre os 6 e 12 anos.

O svalores apresentados por este estudos são similar es com os descritos acima, tanto para as meninas eutróficas $\left(\mathrm{VO}_{2 \max }\right.$ $\mathrm{mL} / \mathrm{min} / \mathrm{kg}=40,23 \pm 6,98)$, quanto paraas com desnutrição pregressa $\left(\mathrm{VO}_{2 \text { max }} \mathrm{mL}\right.$ ) $\mathrm{min} / \mathrm{kg}=40,53 \pm 6,32$ ), eestão de acordo com os valores de consumo máximo de oxigênio previstos por Cooper \& WeilerRavell (1984) para criançasmenores de 13 $\operatorname{anos}(38 \pm 7 \mathrm{~mL} / \mathrm{min} / \mathrm{kg})$.

Emumestudo realizado por Rutenfranzet al. (1981) osvalores médios de consumo de oxigênio parameninasnorueguesascomidade médiadenove anos, expresso emlitros/min, foide $1,48 \pm 4$. Esteestudo apresentouvalores menores para as eutróficas $(1,11 \pm 0,25)$ e para as meninas com desnutrição pregressa $(1,02 \pm 0,19)$. Essadiferençapodeestar associadaadiferençasétnicas, nutricionaise, também, a diferenças de composição corporal, uma vez que a altura média das meninas do citado estudo éde $136,1 \mathrm{~cm}$, enquanto quea desteestudo éde $131,31 \mathrm{~cm}$ paraaseutróficas e125,06 paraascom desnutrição pregressa.
A maioria dos estudos sobre aptidão cardiorrespiratóriarealizadosem crianças brasileiras utilizaram métodos indiretos (Desai et al., 1981; Anjos\& Boileau, 1988; Duarte \& Duarte, 1989), enão apresentaram valores de consumo de oxigênio obtidospor metodologiasemelhanteàutilizada nesteestudo.

Desai etal. (1981) utilizaram afreqüênciacardíacacom determinantedacapacidade de trabalho físico em testes realizados com crianças em bicicletaergométrica. Foi relatado que crianças de baixo nível socioeconômico (bóias-frias) quando comparadasacrianças de classemédiade cidades do interior de São Paulo, apresentaram uma menor performance física, relacionando-a com um menor tamanho corporal e uma menor massa magra, conseqüentes de desnutrição nos primeiros anos de vida. Foi também observado que criançasdesnutridas apresentavam valoressignificativamentemaioresparaFC nasmesmascargas de trabalho de crianças eutróficas, o que sugere que crianças desnutridas apresentaram menor condicionamento físico que controles.

Anjos \& Bo ileau (1988) utilizaram aintensidade de esforço necessáriaparaelevar aFC até 170 bpm (PWC 170) paradeterminação da aptidão cardiorrespiratória em meninoseutróficosedesnutridos com desnutrição vigente. Duarte \& D uarte (1989) avaliaram a capacidade aeróbiade crianças eutróficas de 10 a 18 anos com a aplicação do testePWC 170. Entretanto, Reybrouck; Weymans; Stijns \& Van der Hauwaert (1986), não obtiveram bons resultados com PWC 170 para diferenciar crianças com cardiopatia em relaçãa a um grupo controle sem doença, e concluíram que 0 limiar anaeróbio ventilatório é um índice maissensível.

Barac-N ieto, Spurr, Reina (1984) conduziram um estudo, comparando o consumo de oxigênio de crianças desnutridas e eutróficas, e concluíram que os resultados mostrando que $0 \mathrm{VO}_{2 \max }$ das desnutridas expresso por kgde peso corporal eramaior quedas eutróficas se deviaao fato de que as crianças desnutridas apresentavam maior proporção de massa magra corporal.

Nossos resultados demonstram que meninas com desnutrição pregressa apresentam VO ${ }_{2 \max }$ erespostas cardiorrespiratóriasao exercício máximo similaresàs das meninas eutróficas. A capacidade máxima de realização de trabalho (watts max) durante o teste de esforç̧o máximo também não apresentoudiferenças entreosgrupos. Assim, é provável que a desnutrição nos primeiros anos de vida possanão ter efeito longitudinal seacriançarecuperar arelação peso/estaturaesperada, apósum aumento naingestão calórica.

O svalores de consumo de oxigênio no limiar anaeróbio encontradosnesteestudo são similares aos descritos por Cooper; Weiler-Ravell; Whipp \& Wasserman, (1984), de $23 \pm 4 \mathrm{~mL} / \mathrm{min} / \mathrm{kg}$, e os por Armon; Cooper; Flores; Zanconato \& Barstow, (1991), de 23,6 $53,5 \mathrm{~mL} / \mathrm{min} / \mathrm{kg}$, estão de acordo com os valores de $23 \pm 4$ $\mathrm{mL} / \mathrm{min} / \mathrm{kg}$ previstosparameninasmenores de 13 anos (Cooper \& Weiler-Ravell, 1984).

N este estudo não foi possível determinar diferenças no consumo de oxigênio do limar anaeróbio, expresso por quilograma de peso corporal, entremeninas eutróficas e com desnutrição pregressa. Aparentemente, arecuperação darelação esperada de peso/estaturaé suficiente parao restabelecimento da capacidade de trabalho submáximo.

As diferenças encontradas, entre as meninas com desnutrição pregressa e as eutróficas para os valores de consumo de oxigênio no limiar anaeróbio ventilatório expresso por litros/min e em porcentagem do $\mathrm{VO}_{2 \text { max }}$ porcentagem do $\mathrm{VE}_{\text {max }}$ no limiar anaeróbio ventilatório eVE no limiar anaeróbio ventilatório, parecem estar mais rela- 
Fernandez Ac et al.

cionadas com a diferença de estatura existente, visto que os maiores valores foram apresentadospelas eutróficasque possuem maior estatura que as com desnutrição pregressa.

A inexistênciade diferençassignificativas na carga de trabalho no limiar anaeróbio indicaqueasmeninas apresentam capacidades de realização de trabalho submáximo semelhantes (mesma cargaem watts), mas, quando se considera 0 gasto energético representado por litros de $\mathrm{O}_{2}$ consumido, observa-se que as meninas com desnutrição pregressaparecem apresentar melhor economia de movimento, isto é, menor consumo de oxigênio para uma mesma intensidade detrabalho.

Fatores relacionados com a composição corporal, não analisada no presente estudo, podem ser responsáveis pela meIhor economia de movimento observada no grupo formado por meninas com desnutrição pregressa.

O smenores valores defreqüênciacardíaca e VE, apresentados no limiar anaeróbio ventilatório pelas meninas com desnutrição pregressa, parece indicar umamenor so brecarganossistemas cardiovascular e respiratória paraarealização de trabalho submáximo, porém são necessários estudoscomplementares parauma comprovação maisdetalhadaarespeito dasobrecarga nos sistemas cardiovascular e respiratório durante o exercício em crianças com desnutrição pregressa.

O sdados do presente estudo sugerem que as crianças desnutridas pregressas apresentam uma adaptação para produzir menor estresse fisiológico paraa realização deatividadefísicasubmáxima ou moderada, quando comparadas a crianças eutróficas.

De acordo com os resultados deste estudo, 0 critério de restabelecimento da relação esperada peso/estatura paraconsiderar crianças com desnutrição pregressa como recuperadas parece ser adequado também paraindicar normalidade dapotência aeróbiamáxima, limiar anaeróbio, resposta ventilatória e de freqüênciacardíaca ao exercício.

\section{Summary}

Metabolic and cardiovascular responses to maximal and submaximal exercise in eutrophic and stunted girls

OBjective. The metabolic and cardiovascular responses of eutrophic girls and girls who underwent early undernutrition, were examined during physical exercise, in order to verify whether previous undernutrition could, in addition to cause a deficit in the children's height, and limit their functional capability to perform a maximal and submaximal exercise in an ergometric bicycle.

SuBjECTS. Our sample was composed of 24 girls, of which 12 were eutrophic, with $9.13 \pm 0.79$ years (mean \pm s.d.), $27.64 \pm$ $3.64 \mathrm{~kg}$ of body weight, and $131.31 \pm 6.04$ $\mathrm{cm}$ in height. The 12 early undernourished girls were $9.75 \pm 1.1$ years old, $25.16 \pm$ $2.33 \mathrm{~kg}$, and $125.06 \pm 3.9 \mathrm{~cm}$ high.

MeAsuRements. The method included clinical evaluation and ergo-spirometric test on an ergometric bicycle, from which all the metabolic and cardiac-respiratory variables were obtained and analyzed. The test protocol was an initial two minutes period with 25 watts, followed by increments of 15 watts every two minutes, until exhaustion.

RESULTS. Analysis of the results of the test of maximal effort did not reveal significant differences between eutrophic ( $E$ ) and early undernourished (UN) girls in any of the variables: $\mathrm{VO}_{2 \max }$ expressed in $1 / \mathrm{min}$ ( $\mathrm{E}=$ $1.11 \pm 0.25 ;$ UN $=1.02 \pm 0.19) ; V_{2 \max }$ expressed in $\mathrm{ml} / \mathrm{min} / \mathrm{kg}(E=40.23 \pm 6.98$; $U N=40.53 \pm 6.32), V E_{\max }(E=40.61 \pm$ 8.79; UN $=38.25 \pm 8.95), R(E=1.13 \pm$ $0.07 ; U N=1.13 \pm 0.05), H R_{\text {max }}(E=$ $195.33 \pm 9.13 ;$ UN $=193.85 \pm 11.18$ ), previous \% HR $R_{\max }(E=92.87 \pm 4.33$; UN $=92.80 \pm 4.87)$, and maximum watts $(E=$ $90.00 \pm 20.56$; UN = 91.25 \pm 11.33 ). Analysis of the results obtained in test of submaximal effort, reflected by the intensity of effort in the anaerobic threshold (AT), showed differences between the groups in the following variables: $\mathrm{VO}_{2 \max }$ expressed in $\mathrm{I} / \mathrm{min}$. $(\mathrm{E}=0.77 \pm 0.14 ;$ UN $=0.62 \pm$ $0.13), \%$ of $\mathrm{VO}_{2 \max }(E=70.07 \pm 7.52$; UN $=61.21 \pm 9.83), H R(E=166.58 \pm$ $14.25 ; \mathrm{UN}=146.33 \pm 17.40), \% H R_{\max }(E$ $=85.31 \pm 6.41 ; \mathrm{UN}=75.50 \pm 7.73), \mathrm{VE}$ $(E=24.18 \pm 5.81 ; U N=17.69 \pm 4.30)$, $\% V E_{\max }(E=59.64 \pm 8.45 ; U N=46.95$ \pm 10.59 ). No differences were found for $\mathrm{VO}_{2 \text { max }}$ expressed in $1 / \mathrm{min} / \mathrm{kg}$ ( $E=28.06 \pm$ 4.87; UN $=24.81 \pm 5.31)$ and watts $(E=$ $51.25 \pm 17.47 ;$ UN $=45.00 \pm 9.77$ )

CONCLUSION. The reestablishment of the ratio weight/height in early undernourished girls is also useful to indicate lack of limitation of functional capability to perform physical exercises. [Rev Ass M ed Bras; 46(4): 312-9]

KEY-wORDS: Exercise. 0 xygen consumption. Stunted. Eutrophicgirl.

\section{Agradecimentos}

Flávio Ascanio Antônio Lauro e Sérgio Garcia Stella pela ajuda na realização dos testes e Luciane Grillo por trazer os voluntários para a pesquisa.

\section{Referências Bibliográficas}

1. American College O fSports Medicine (Acsm); Prova de Esforço e Prescrição de Exercícios; Livraria e Editora Revinter Ltda; 1994.

2. Anjos, L. A. And Boileau, R. A. - Performance de garotos desnutridos e não desnutridos em determinados testesfísicos. Revista Brasileira de Ciência e M ovimento, 1988; 2: 21-29;

3. Armon, Y.; Cooper, D.m.; Flores, R.; Zanconato, S.; Barstow, T.J. - O xygen uptake dynamics during high-intensity exercise in children and adults. J. Appl. Physiol., 1991; 70:841-8.

4. Armstrong, N . \& W esman, J.r. - Assessment and Interpretation of Aerobic Fitness in Children and Adolescent; Exerc. Sport. Sci. Rev., 1994; 22: 435-76. 
5. Avelar, J.a.; Margarita, V.r.; Artaza, C.b. C recimiento después de $D$ esnutrición grave precoz. Rev. Child. Pediatr. ; 1991, 62: 242247.

6. Barac-nieto, M.; Spurr, G.b.; Reina, J.c. - Marginal malnutrition in school-aged Colombian boys: body composition and maximal 02 consumption. Am. J. Clin. Nutr. 1984; 39: 830-839.

7. Chaves, N . - N utrição Básica e Aplicada; 2 ed., Rio de Janeiro, Guanabara Koogan, 1985.

8. Cooper, D.m,; W eiler-ravell, D.; W hipp, B.j.; W asserman, K. - Aerobic parameters of exercise as a function of body size during grow th in children. J. Appl. Physiol., 1984; 56:628-34.

9. Cooper, D.m. \& W eiler-ravell, D. - Gas exchange response to exercise in children; Am. Rev. Respir. Dis., 1984; 129 (suppl.): s47-s48,.

10. Desai, I.D.; Tavares, M.I.G.; O liveira, B.S.D .; Desai, M.I.; Romero, L.S.C.; Vichi, F.I.; Duarte, F.a.m.; O liveira, J.e.d. - Anthropometric and cycloergometric assessment of the nutritional status of the children of agricultural migrant workers in Southern Brazil. Am. J. Clin. N utr. 1981; 34:1925-1934.

11. Duarte, C.r. \& Duarte, M.f.s. - Aerobic capacity amongstudents from 10 to 18 years old; Braz. J. Sci. Mov., 1989; 3: 17-25.

12. França, N.M., Matsudo, V.K.R., França,N .M. Skinfold measurement of schoolchildren from 7 to 8 years old, Braz. J. Sci. M ov., 1988; 2: 7 16.

13. Greulich, W. W. - Growth of children of the same race under different environmental conditions. Science, 1958; 127:515-516.

14. Grillo, L.P. - Caraterização das condições metabólicas, nutricionais e sócio-econômicas de meninas escolares eutróficas e desnutridas pregressas moradoras em favelas do município de São Paulo. São Paulo, 1996 [Tese - Mestrado - U niversidade Federal de São Paulo EPM]

15. Meredith, C.N . E D wyer, J.T. - N utrition and Exercise: Effects on Adolescent H ealth; Annu. Rev. Public. H ealth; 1991;12:309-33.

16. $\mathrm{N}$ ational Center For Health Statistics (N CHS). Plan and operation of the health and nutrition examination survey. 1973; USA 1971-73. Vital $\mathrm{H}$ ealth Stat 1. n.10a and $10 \mathrm{~b}$.

17. Pesquisa $N$ acional Sobre Saúde e N utrição (PN SN ) - Arquivos dos dados da pesquisa IN AN , Brasília, 1989.

18. Reybrouck, T.; W eymans, M.; Stijns, H .; Van Der Hauwaert, L.G. - ventilatory anaerobic threshold for evaluating exercise performance in children with congenital left-to-right intracardiac shunt. Pediatr. Cardiol., 1986; 7: 21.

19. Rutenfranz, J.; Andersen, K.I.; Seliger, V.; Klimmer, F.; Berndt, I.; Ruppel, M - - Maximal aerobic power and body composition during the puberty growth period: similarities and differences between children of two European countries. Eur. J. Pediatr. 1981; 136: 123-133.

20. Satyanarayana, K.; Krishna, T.p.; Rao, B.s.n. Effect of early childhood undernutrition and child labor on growth and adult nutritional status of rural Indian boys around $\mathrm{H}$ yderabad. H um. Nutr. Clin. N utr. ; 1986, 40C:131-139.

21. Sawaya, A.I.; D allal,G.; Solymos, G.; De Sousa, M.h.; Ventura, M.l.; Roberts, S.b.; Sigulem, D.m. - $O$ besity and Malnutrition in a Shantytown Population in the City of São Paulo, Brazil; Obs. Res., 3 (Suppl. 2) 1995;107s$115 \mathrm{~s}$.

22. Sawaya,A.l.; Amigo, H.; Sigulem, D.M. - Alterações metabólicas e antropométricas na desnutrição proteico-calórica em crianças do município de São Paulo;J. Pediatr.; ; 1989, 65: 393-98.
23. Siegel, S. - Estatisitica no parametrica; Ed. Trillas; México; 1975.

24. Sokal, R.r. \& Rohlf, F. J. - Biometry; W. H. Freeman and Company; San Francisco; 1969.

25. Spurr,G.b. E Reina,J.c. - Daily pattern of $\%$ VO 2máx. and heart rates in normal and undernourished school children; Med. Sci. Sport Exerc., 1990; 22: 643-652,.

26. Spurr,G .b.; Reina, J.c.; Dahners, H.w.; Baracnieto, M. - Marginal malnutrition in schoolaged Colombian boys: functional consequences in maximum exercise; Am. J. Clin. N utr., 1983; 37:834-47.

27. Waterlow, J.C. - The presentation, and use of height and weight data for comparing the nutritional status of groups of children under the age of 10 years. Bull. W orld H ealth 0 rgan., 1977; 55: 489-498.

28. W aterlow, J.C.; - C lassification and definition of protein - energy malnutrition; In: Beaton, G.h.; Bengoa, J.m.; eds. N utrition in preventive medicine; the major deficiency syndromes, epidemiology and approaches to control, Geneva; W old Healt O rganization; 1976, $p$. 530-555.

29. Zeballos, R.J. \& W eisman, I.M. - Behind the scenes of cardiopulmonary exercise testing. In: W eisman, I.M. \& Zeballos, R.J.; Clinics in chest medicine: clinical exercise testing; W.B. Saunders Company; v. 15; n.2; 1994, p.193-214.

Artigo recebido: 21/07/98 Aceito para publicação: 15/08/00 